\title{
Learning to deal with dual use
}

\section{Megan J. Palmer}

Megan J. Palmer is a senior research scholar at the Center for International Security and Cooperation (CISAC) at Stanford University, Stanford, CA, USA. mjpalmer@stanford.edu

Biological research is profoundly valuable but can carry profound risks. The coronavirus outbreak reminds us of our vulnerability to biological threats and that research on pathogens is vital to threat mitigation. But such research can lead to catastrophic safety and security incidents. A global proliferation of tools and capabilities, driven by economic and national security interests, is also generating risks that fall outside current governance frameworks. We must learn to manage risks as quickly as we learn to manipulate life, but it remains unclear how well we are doing. One opportunity to learn is found in a new charge to the U.S. National Science Advisory Board for Biosecurity (NSABB), which reconvened in January after a 2-year hiatus, to assess the effectiveness of U.S. dual-use research oversight policies. To meet the charge and suggest improvements, the NSABB must address a neglected need for an evidence base for adaptive risk management. Building this evidence base will require revisiting success criteria, creating data infrastructures, and fostering adaptive policies and testbeds.

Clear criteria for success form the foundation of an evidence base. Criteria for policies designed to prevent rare incidents can be challenging to develop, but there are leading indicators that can validate design goals and assumptions. These can range from enhanced stakeholder participation and expressions of trust to reductions in near misses. Such criteria form the basis for sound policy design and implementation and must be regularly revisited.

The next step is to collect and analyze data to assess whether goals were met. A 2011 U.S. government-commissioned risk and benefit analysis of gain-of-function research exposed the dearth of data available to inform many risk management decisions. The NSABB and other groups have called-to no avail-for systems to collect and analyze data on incidents and near-misses as well as oversight. Failure of the U.S. government to quickly act on these recommendations means that the NSABB will again confront this data gap. Moreover, biosafety and biosecurity research, which seeks to understand and improve practices for risk management, remains chronically underfunded despite being called out as a priority in the U.S. National Biodefense Strategy. This research should be supported as an essential part of all major biological research programs so that it evolves alongside and is integrated with the latest advances.

Unfortunately, even when limited data are collected, they are often not shared lest they expose vulnerabilities or tarnish reputations. Legitimate security concerns abound, but an unknown and growing risk exposure is also dangerous. Many organizations do not fully exercise their ability to share details of their risk management process; they should do so to promote cross-organizational learning and to avoid repeating the same mistakes.

Policies designed to adapt to emerging evidence can provide standards and incentives for gathering data. Yet dualuse policies are typically narrowly scoped to prioritize limiting oversight burden over learning where concerns arise. This priority is misplaced in preparing for the future. As the scope of research broadens, our definitions of dual use will change, and we need to continue moving toward functional definitions of activities that could be hazardous that aren't reliant on quickly outdated lists. In many instances where there is uncertainty over appropriate scope, we should update policies regularly and prioritize observing activities over restricting them to gain visibility into the evolving landscape.

Communities of practitioners need testbeds to experiment with policy approaches and to learn from each other in real time. One example is the international Genetically Engineered Machine (iGEM) competition, where thousands of students from dozens of countries work on hundreds of projects that can often expose policy gaps. The competition has becomes a nexus to test strategies for managing dual-use technologies across many cases with yearly iteration. Such testbeds should be cultivated at many scales-from individual labs, to universities, to regional and national networks.

Biological science and technology will only become more essential to our societies, and we need strategies to learn to manage their power. Investing in an evidence base for adaptive risk management is essential to ensure that the future of the life sciences is one in which we want to live.

Published online 27 February 2020
10.1126/science.abb1466 